\title{
On nonisometric isospectral connected fractal domains
}

\author{
Brian D. Sleeman and Chen Hua
}

\begin{abstract}
A fundamental question raised by M. Kac in 1966 is: Must two isospectral planar domains necessarily be isometric? Following a long history of investigation C. Gordon, D. L. Webb and S. Wolpert in 1992 finally proved that the answer is no. By using the idea of transposition maps one can construct a wide class of planar domains with piecewise continuous boundaries which are isospectral but nonisometric. In this note we study the Kac question in relation to domains with fractal boundaries and by following a technique of paper folding maps by J. Chapman 1993 we construct pairs of isospectral but nonisometric connected planar domains with fractal boundaries having the same Minkowski dimension.
\end{abstract}

\section{Introduction.}

Let $(M, g)$ be a compact Riemann manifold with boundary. Then $M$ has a Laplace operator $\Delta$ defined by

$$
\Delta f=-\operatorname{div}(\operatorname{grad} f)
$$

that acts on functions defined on $M$. The spectrum of $M$ is a sequence of eigenvalues on $\Delta$. Two Riemann manifolds are isospectral if their spectra (counting multiplicities) coincide. A fundamental question concerning the relationship between spectral analysis and geometry raised 
by Kac [1] is: must two isospectral planar domains necessarily be isometric? For Riemann manifolds the answer to this question is no in general as shown through a wide range of ingeneously constructed examples by Milnor [2], Vigneras [3], Buser [4], Brooks [5], Brooks and Tse [6], Ikeda [7], Urakawa [8], Gordon and Wilson [9], and De Turck and Gordon [10]. However Kac's question concerning planar domains remained unresolved until the ground breaking work of Gordon, Webb and Wolpert [11], [12] in 1992 in which the answer was once again a resounding NO. Furthermore their work allows one to construct a whole variety of pairs of isospectral, non-isometric planar domains. One such pair is that shown in Figure 1.

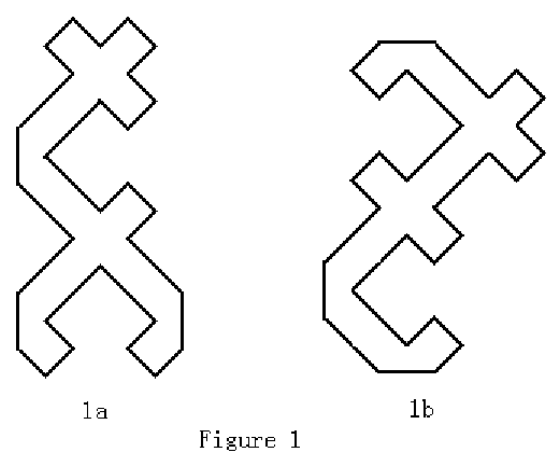

Following Gordon.et.al., Berard [13], [14] has given a simple proof showing that the eigenvalues of Figure 1a are identical to those of Figure 1 b by constructing a map which takes an eigenfunction of the first domain and maps it onto an eigenfunction for the second domain with the same eigenvalue.

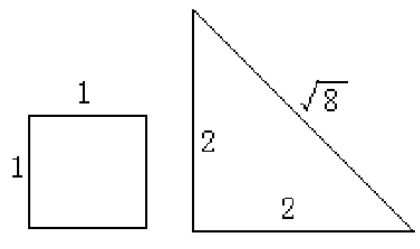

$2 \mathrm{a}$

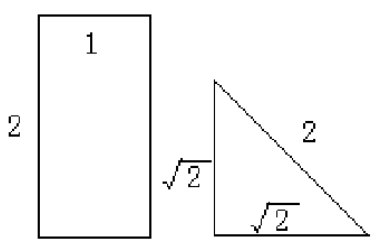

$2 b$

Figure 2

This idea of transposition maps has been developed further by Buser.et.al. [15] to generate new examples of isospectral domains. Furthermore Chapman [16], [17] has given a pair of disjoint isospectral domains for which the eigenvalues can be explicitly calculated. These 
domains are shown in Figure 2.

By separation of variables methods or otherwise it is easy to show that the eigenvalues for Figure $2 \mathrm{a}$ are the sets

$$
\left\{\left(n^{2}+m^{2}\right) \pi^{2}\right\} \cup\left\{\left(\frac{i^{2}}{4}+\frac{j^{2}}{4}\right) \pi^{2}\right\}, \quad n, m, i, j \in \mathbb{N}, i>j
$$

while those for Figure $2 b$ are

$$
\left\{\left(\frac{N^{2}}{4}+M^{2}\right) \pi^{2}\right\} \cup\left\{\frac{1}{2}\left(I^{2}+J^{2}\right) \pi^{2}\right\}, \quad N, M, I, J \in \mathbb{N}, I>J
$$

All the above results and ideas relate to domains which are at least piecewise smooth. In view of the current interest in fractal geometry [18] and its important applications in science it is natural to ask similar questions about domains which are fractal in some sense. In [19] the authors have taken up this question and have explicitly constructed a class of disjoint domains with fractal boundaries which are non-isometric and isospectral with boundaries which have the same interior Minkowski dimension. Levitin-Vassiliev [20] has also demonstrated the possibility of non-isometric but isospectral disjoint fractal domains but gave no examples. The domains constructed in [19] are based on a tessellation of $\mathbb{R}^{2}$ of various arrangements of the isospectral pairs shown in Figure 2. Furthermore it is shown how explicit two-term asymptotics may be determined for the associated spectral counting functions.

In this paper we develop the problem and construct non-isometric isospectral drums which are connected but have fractal boundaries. The key to these constructions is a "paper folding" argument due to Chapman [17] which is a constructive method of realising the transposition maps of Berard [13], [14] and Buser.et.al. [15].

In Section 2 we describe the paper folding technique of Chapman [17] and use it to construct a pair of non-isometric, isospectral domains based on the 30, 60 right triangle. Section 3 provides a construction of connected non-isometric, isospectral domains with fractal boundaries.

\section{Paper Folding Maps.}

In this section we describe the idea of transposition (paper folding) maps to prove that the two domains shown in figure 3 composed of 30 , 60 , right triangles are non-isometric but isospectral. 
To begin with we introduce some notions. Suppose we have a pair of identical triangles such as $A$ and $B$ in Figure $3 \mathrm{a}$. Then $A+B$ means that to obtain the value of a function in that triangle we add the values of the function at the corresponding points in triangles $A$ and $B$. If we need to reflect a triangle about its line of symmetry we indicate this by $\bar{A}$. Only the zero function maps to the zero function. Thus in terms of eigenfunctions for any eigenfunction of the eigenvalue problem for $A$ there is a corresponding eigenfunction of $B$ for the same eigenvalue.

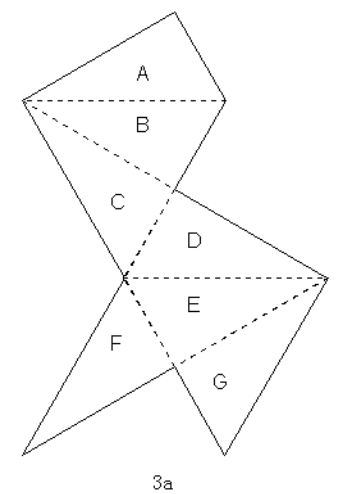

Figure 3

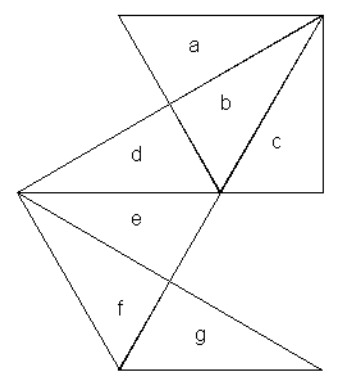

The paper folding idea proceeds as follows: Consider the domain in Figure $3 \mathrm{a}$ and fold it along the dotted line separating the domains $E$ and $G$ and $E$ and $D$ to get the domain shown in Figure 4

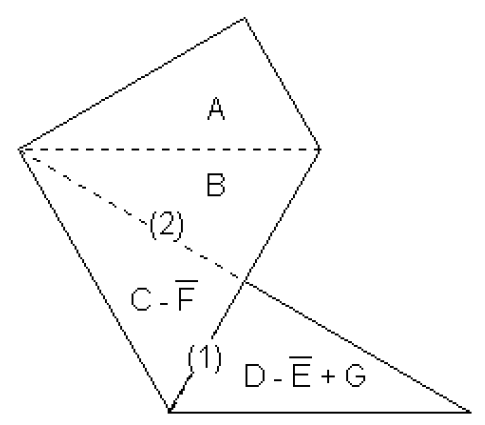

Figure 4

We then obtain a function which is zero on the boundary. In general we take several copies of the original domain (Figure 3a) and fold them to create new domains. These are then glued together to create a new domain and define the transposition on this domain to be the sum of the transpositions on the composite domains. If we glue the composite domains together in such a way that the first derivatives of the 
transposition is continuous then we will have created an eigenfunction on the new domain.

In order for the first derivative of the transposition to be continuous it is sufficient that;

1) Every fold lies along an outer edge of the new shape.

2) Each edge of each copy of the original shape that lies in the interior of the final shape must be adjacent to its reflection on an associated copy of the original shape.

In Figure 4, the first derivative of the transposition is discontinuous across the lines (1) and (2). However if we add the same initial domain (Figure 3a) folded as in Figure 5 then we ensure continuity of the first derivative across the lines (1) and (2) as shown in Figure 6. However the first derivative is now discontinuous across other lines.

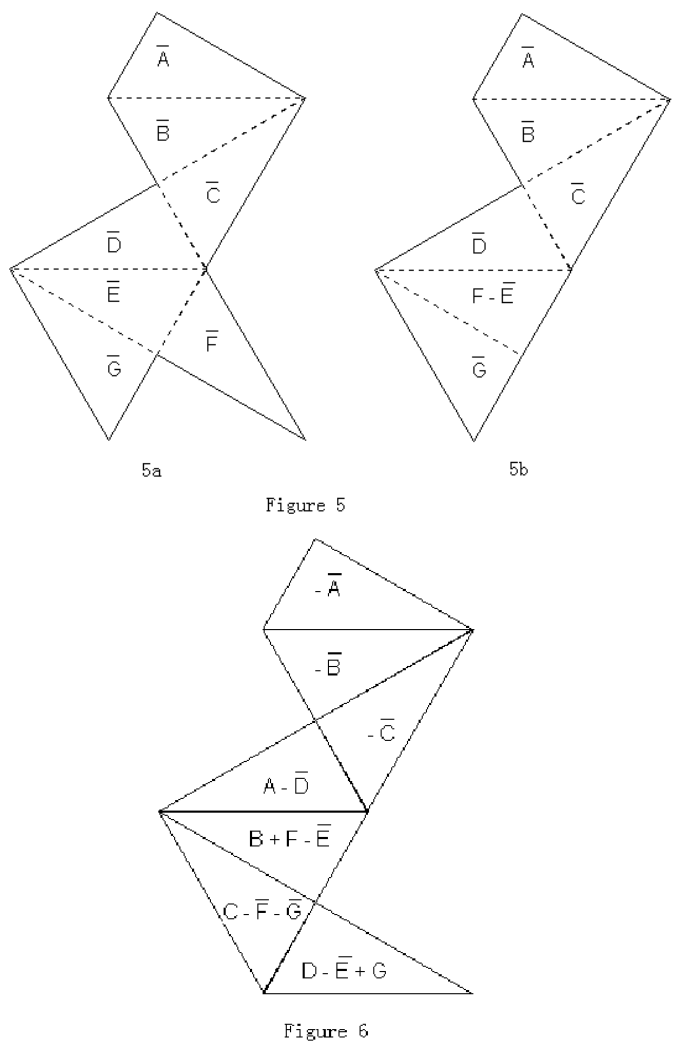

To achieve continuity across all lines we fold Figure 5 along the dotted line separating regions $\bar{D}$ and $\bar{C}$ and then along the dotted line 
separating regions $\bar{B}$ and $\bar{C}$ to obtain Figure 7

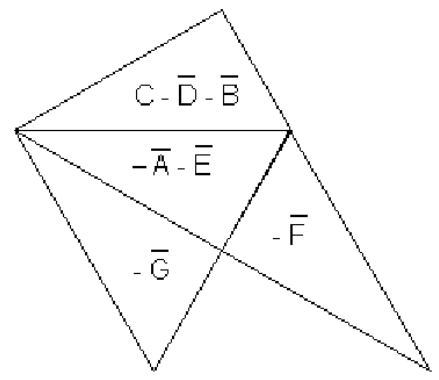

Figure 7

This is then added to Figure 6 to obtain the final shape

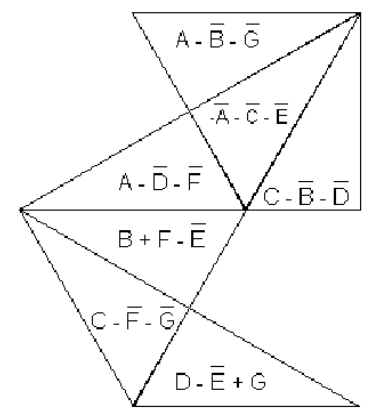

Figure 8

Figure 8 has continuity of derivatives across all interior lines and is the required non-isometric, isospectral domain as claimed in Figure $3 \mathrm{~b}$.

For further details concerning the paper folding technique and other examples see Chapman [17].

\section{Non-Isometric Domains with Fractal Boundaries.}

We have constructed in Section 2 a pair of non-isometric isospectral domains based on the 30,60, right triangle; other base shapes may be used however. Indeed the example of [12] is based on the right angled isosceles triangle; Chapman [17] gives several other examples. It is interesting to note however that if we apply the same construction as in Section 2 based on the 60,60, 60 isosceles triangle then again we obtain a pair of isospectral domains which are also isometric. That is one domain is merely a rotation of the other. It is not clear why this 
should be so; but may be due to the symmetry of the base shape.

Furthermore, it is not necessary for the base shape to be a triangle. Any shape with at least three edges will do. We simply choose three edges to represent the three sides of the triangle about which one reflects the shape. If we then follow the same pattern of reflections that created the shapes of Section 2 we shall again obtain new isospectral domains. $[17$, Figure 15] constructs quite exotic shapes. It is this observation that allows us to construct isospectral domains with fractal boundaries.

Consider the base triangle used in Section 2 as shown in Figure 9 modified with the shaded region

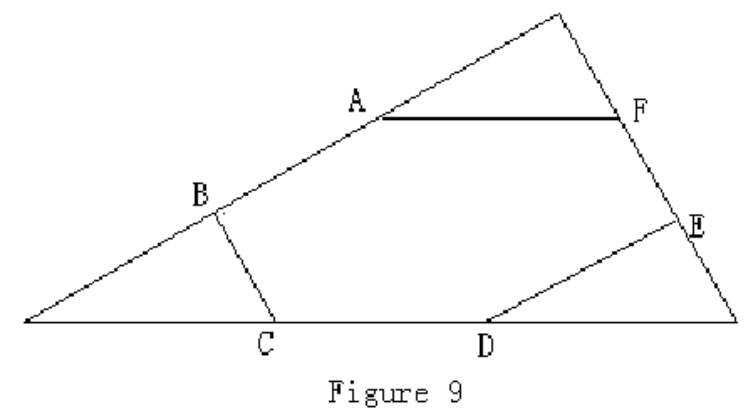

On the edges $A F, E D$ and $B C$ of the shaded region we construct in the usual way appropriately scaled segments of the Koch curve based on the standard triadic Cantor set as shown in Figure 10.

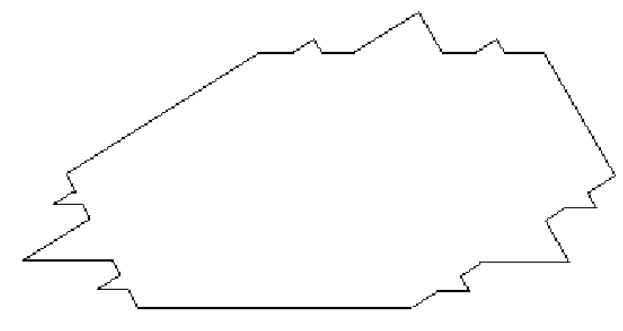

Figure 10

The next step is to impose this structure on the isospectral domains of Section 2. This results in the Figures 11.

Actually the domains in Figure 11 are connected domains with fractal boundaries as shown in Figure 12.

Clearly Figures $12 \mathrm{a}$ and $12 \mathrm{~b}$ are non-isometric. That they are also isospectral follows from the weak formulation of the Dirichlet eigenvalue problem as discussed in [22] and the references given there. Furthermore 
it is easy to see that the interior Minkowski dimension of the boundary of these non-isometric isospectral domains is $\delta=\log 4 / \log 3$ (cf. [18]).

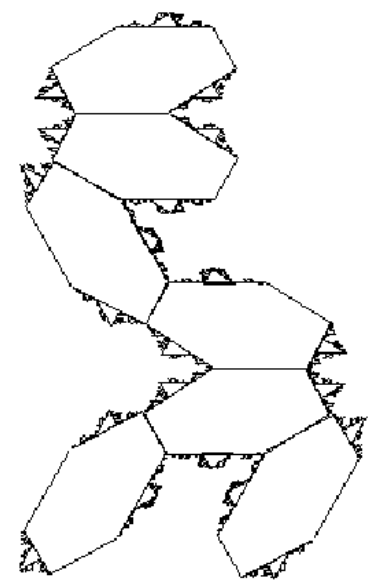

$11 \mathrm{a}$

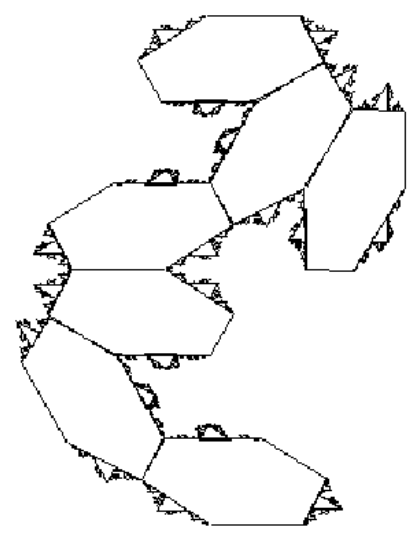

$11 \mathrm{~b}$

Figure 11
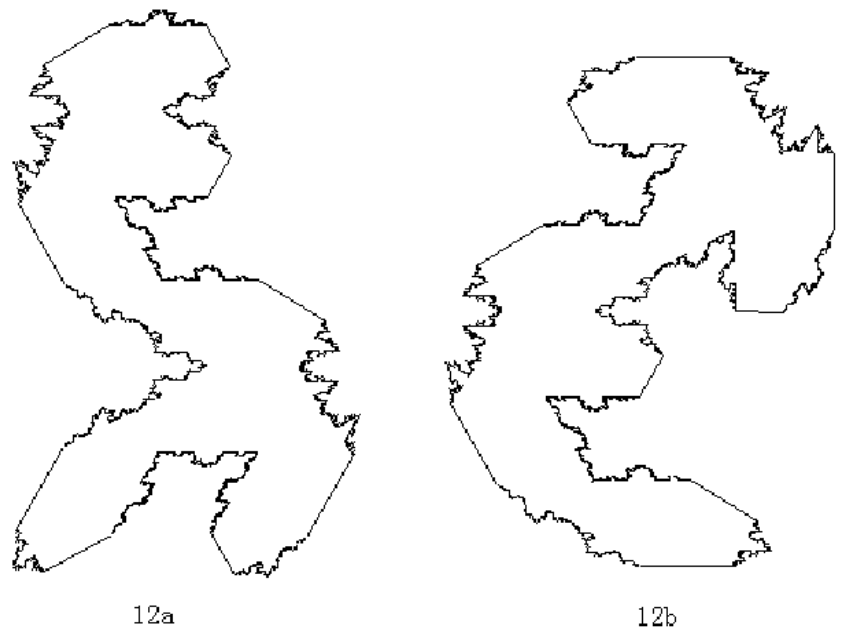

Figure 12

For this particular example (the boundary is quasicircle) we note that the same procedure works if the Dirichlet boundary condition is replaced by the Neumann boundary condition. The only difference in the construction being that where we subtract reflected triangles we now add them. Thus the isospectral domains constructed previously are also isospectral with the new boundary condition (also see [17]). 
For more general fractal boundaries, we need to ensure that the Neumann Laplace operator has only eigenvalues as its spectrum. For this to be the case it is sufficient that the boundaries of our fractal domains satisfy the so-called C'-condition; see [24].

As with the domains of Section 2 and those of this section we may discuss the associated problem of studying the counting function asymptotics of the counting function $N(\lambda)$ inspired by the Weyl-Berry conjecture. We do not go into this here but cite the following works on this subject of current intensive activity [21]-[24]

In another direction it is of interest to ask whether there exists a pair of isospectral but not isometric fractal domains. By a fractal domain $\Omega$ we mean a domain in which $\Omega$ itself is fractal as opposed to the situation treated in this note in which the boundary $\partial \Omega$ is fractal. One of the most familiar examples is the so called Sierpinski gasket ( $c f$. [18]). This problem is significantly distinct from that treated here in that we need to define the laplacian appropriately on such a domain as well as associated eigenvalues. One also needs to define appropriate transposition maps. For a discussion of the construction of Laplacians on fractal lattices and associated counting function asymptotics we refer to Kigami and Lapidus [25] and the references cited there. This problem will be the subject of further work.

Acknowledgements. The main ideas for this note were initiated in 1994 when B. D. Sleeman visited the Department of Mathematics, Wuhan University.

Both authors are indebted to David Sleeman for computing the figures.

\section{References.}

[1] Kac, M., Can one hear the shape of a drum? Amer. Math. Monthly 73 (1966), 1-23.

[2] Milnor, J., Eigenvalues of the Laplace operator on certain manifolds. Proc. Nat. Acad. Sci. USA 51 (1964), 542.

[3] Vigneras, M. F., Variete's Riemannienes isospectrales et non isometriques. Ann. of Math. 112 (1980), 21-32.

[4] Buser, P., Isospectral Riemann surfaces. Ann. Inst. Fourier 36 (1986), 167-192. 
[5] Brooks, R., Constructing isospectral manifolds. Amer. Math. Monthly 95 (1988), 823-839.

[6] Brooks, R., Tse, R., Isospectral surfaces of small genus. Nagoya Math. J. 107 (1987), 13-24.

[7] Ikeda, A., On lens spaces which are isospectral but not isometric. Ann. Sci. Ecole Norm. Sup. 4 (1980), 303-315.

[8] Urakawa, H., Bounded domains which are isospectral but not congruent. Ann. Sci. Ecole Norm. Sup. 4 (1982), 441-456.

[9] Gordon, C., Wilson, E., Isospectral deformations of compact solvmanifolds. J. Differential Geom. 19 (1984), 241-250.

[10] De Turck, D., Gordon, C., Isospectral deformations 1, Riemann structures on two-step nilspaces. Comm. Pure Appl. Math. 40 (1987), 367-387.

[11] Gordon, C., Webb, D. L., Wolpert, S., One cannot hear the shape of a drum. Bull. Amer. Math. Soc. 27 (1992), 134-138.

[12] Gordon, C., Webb, D. L., Wolpert, S., Isospectral plane domains and surfaces via Riemannian orbifolds. Invent. Math. 110 (1992), 1-22.

[13] Berard, P., Transplantation et isospectralite I. Math. Ann. 292 (1992), 547-559.

[14] Berard, P., Transplantation et isospectralite II. J. London Math. Soc. 48 (1993), 565-576.

[15] Buser, P., Conway, J., Doyle, P., Semmler, K-D., Some planar isospectral domains. Internat. Math. Res. Notices. 9 (1994), 391ff approx 9pp (electronic).

[16] Chapman, J., You can't always hear the shape of a drum. What's Happening in the Mathematical Sciences. 1 (1993), 13-16.

[17] Chapman, J., Drums that sound the same. Amer. Math. Monthly 102 (1995), 124-138.

[18] Falconer, K., Fractal geometry. John Wiley and Sons, 1990.

[19] Sleeman, B. D., Chen, H., On a pair of non-isometric isospectral domains with fractal boundaries and the Weyl-Berry conjecture. Chin. Ann. of Math. 19 (1998), 9-20.

[20] Levitin, M., Vassiliev, D., Some examples of two-term spectral asymptotics for sets with fractal boundary, Partial differential operators and mathematical physics. Holzhau (1994), 227-233.

[21] Levitin, M., Vassiliev, D., Spectral asymptotics, Renewal theorem and the Berry conjecture for a class of fractals. Proc. Lond. Math. Soc. 72 (1996), 188-214.

[22] Chen, H., Sleeman, B. D., Fractal drums and the $n$-dimensional modified Weyl-Berry conjecture. Comm. Math. Phys. 168 (1995), 581-607. 
[23] Molchanov, S., Vainberg, B., On spectral asymptotics for domains with fractal boundaries. Comm. Math. Phys. 183 (1997), 85-117.

[24] Lapidus, M. L., Vibrations of fractal drums, the Riemann hypothesis, waves in fractal media and the Weyl-Berry conjecture. Proc. Dundee Conference on "Ordinary and partial differential equations". IV (1993), 126-209.

[25] Kigami, J., Lapidus, M. L., Weyl's problem for the spectral distribution of Laplacians on P. C. F. self-similar fractals. Comm. Math. Phys. 158 (1993), 93-125.

Recibido: 26 de octubre de 1.999

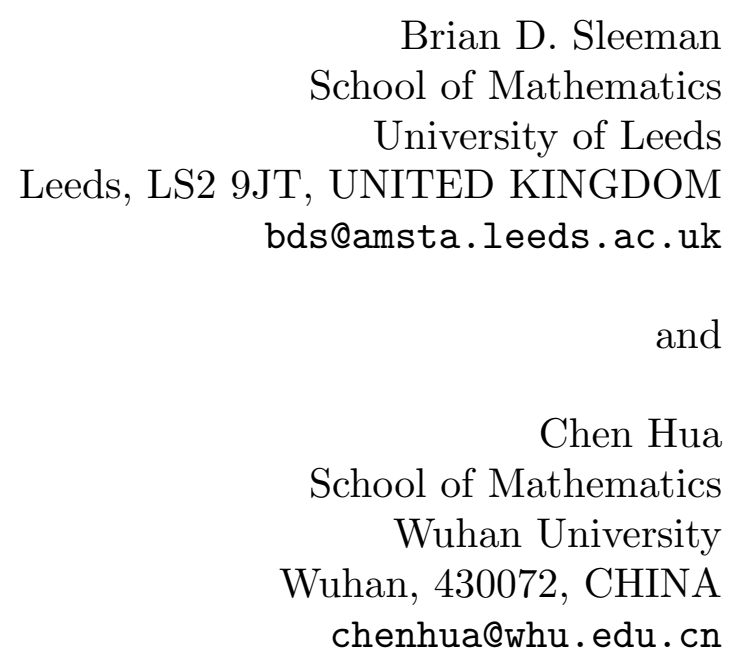

Supported by the Royal Society of London and the Natural Science Foundation of China 